\title{
Approximately algebraic tensor products
}

\author{
Ismail Nikoufar and Themistocles M. Rassias
}




\title{
APPROXIMATELY ALGEBRAIC TENSOR PRODUCTS
}

\author{
ISMAIL NIKOUFAR AND THEMISTOCLES M. RASSIAS
}

Received 23 May, 2014

\begin{abstract}
Let $X$ and $Y$ be normed spaces over a complete field $\mathbb{F}$ with dual spaces $X^{\prime}$ and $Y^{\prime}$ respectively. Under certain hypotheses, for given $x \in X, y \in Y$ and a mapping $u$ from $X^{\prime} \times Y^{\prime}$ to $\mathbb{F}$, we apply Hyers-Ulam approach to find a unique bounded bilinear mapping $v$ near to $u$ such that $\|v\|=\|x \otimes y\|$.
\end{abstract}

2010 Mathematics Subject Classification: 46B10; 47L50; 39B72

Keywords: algebraic tensor product, dual space, stability

\section{INTRODUCTION}

Let $X, Y$, and $Z$ be normed linear spaces over the same field $\mathbb{F}$. A mapping $\phi$ : $X \times Y \longrightarrow Z$ is said to be bilinear if the mappings $x \longmapsto \phi(x, y)$ and $y \longmapsto \phi(x, y)$ are linear. A bilinear mapping $\phi: X \times Y \longrightarrow Z$ is said to be bounded if there exists $M>0$ such that $\|\phi(x, y)\| \leq M\|x\|\|y\|$ for all $x \in X$ and $y \in Y$. The norm of $\phi$ is then defined by

$$
\|\phi\|:=\sup \left\{\|\phi(x, y)\|:(x, y) \in \mathscr{B}_{X} \times \mathscr{B}_{Y}\right\},
$$

where $\mathscr{B}_{X}:=\{x \in X:\|x\| \leq 1\}$. The set of all bounded bilinear mappings from $X \times Y$ to $Z$ is denoted by $\mathscr{B} \mathscr{L}(X \times Y, Z)$. Let $X^{\prime}$ and $Y^{\prime}$ be dual spaces of $X$ and $Y$ respectively. For given $x \in X$ and $y \in Y, x \otimes y$ is an element of $\mathcal{B} \mathscr{L}\left(X^{\prime} \times Y^{\prime}, \mathbb{F}\right)$ defined by $x \otimes y(f, g):=f(x) g(y)$ for all $f \in X^{\prime}$ and $g \in Y^{\prime}$. The algebraic tensor product of $X$ and $Y, X \otimes Y$, is defined to be the linear span of $\{x \otimes y: x \in X, y \in Y\}$ in $\mathscr{B} \mathscr{L}\left(X^{\prime} \times Y^{\prime}, \mathbb{F}\right)$ (see [3]).

A classical question in the theory of functional equations is the following (see [4], [6], [7], [9], [10], [8], [12], [14], [15], [20], [19], [17], [18], [21], [13], [22]): When is it true that a function which approximately satisfies a functional equation $\zeta$ must be close to an exact solution of $\zeta$ ?

If the problem accepts a solution, we say that the equation $\zeta$ is stable. There are cases in which each approximate solution is actually a true solution. In such cases, we call the equation $\zeta$ superstable. 
The first stability problem concerning group homomorphisms was raised by Ulam [22] during his talk before a Mathematical Colloquium at the University of Wisconsin in 1940. Ulam's problem was partially solved by Hyers [7] for mappings between Banach spaces. Hyers' Theorem was generalized by Aoki [1] for additive mappings and by Th. M. Rassias [16] for linear mappings by considering an unbounded Cauchy difference. The paper of Th. M. Rassias [16] has provided a lot of influence in the development of what is called the generalized Hyers-Ulam stability or the Hyers-Ulam-Rassias stability of functional equations. A generalization of the Th. M. Rassias theorem was obtained by Gavruta [5] in 1994 by replacing the unbounded Cauchy difference by a general control function in the spirit of Th. M. Rassias' approach. Badora [2] proved the generalized Hyers-Ulam stability of ring homomorphisms, which generalizes the result of D. G. Bourgin. Miura [11] proved the generalized Hyers-Ulam stability of Jordan homomorphisms.

In this paper, under certain hypotheses and using Hyers-Ulam approach, we find a unique bounded bilinear mapping $v$ near to a given mapping $u: X^{\prime} \times Y^{\prime} \longrightarrow \mathbb{F}$ such that $\|v\|=\|x \otimes y\|$ for $x \in X, y \in Y$. Throughout this paper, it is assumed that $X$ and $Y$ are normed spaces over a complete field $\mathbb{F}$ with dual spaces $X^{\prime}$ and $Y^{\prime}$ respectively.

\section{Results}

Theorem 1. Let $u: X^{\prime} \times Y^{\prime} \rightarrow \mathbb{F}$ be a mapping for which there exist positive real valued functions $\varphi_{1}, \varphi_{2}$, and $\varphi$ on $X^{\prime} \times X^{\prime} \times Y^{\prime}, X^{\prime} \times Y^{\prime} \times Y^{\prime}$, and $X^{\prime} \times Y^{\prime}$, respectively such that

$$
\begin{gathered}
\tilde{\varphi}(f, g):=\sum_{i=0}^{\infty} \frac{1}{2^{i+1}} \varphi_{1}\left(2^{i} f, 2^{i} f, g\right)<\infty \\
\lim _{n \rightarrow \infty} \frac{1}{2^{n}} \varphi_{1}\left(2^{n} f_{1}, 2^{n} f_{2}, g\right)=\lim _{n \rightarrow \infty} \frac{1}{2^{n}} \varphi_{2}\left(2^{n} f, g_{1}, g_{2}\right)=\lim _{n \rightarrow \infty} \frac{1}{2^{n}} \varphi\left(2^{n} f, g\right)=0 \\
\left|u\left(c f_{1}+f_{2}, g\right)-c u\left(f_{1}, g\right)-u\left(f_{2}, g\right)\right| \leq \varphi_{1}\left(f_{1}, f_{2}, g\right) \\
\left|u\left(f, c g_{1}+g_{2}\right)-c u\left(f, g_{1}\right)-u\left(f, g_{2}\right)\right| \leq \varphi_{2}\left(f, g_{1}, g_{2}\right)
\end{gathered}
$$

for all $f, f_{1}, f_{2} \in X^{\prime}, g, g_{1}, g_{2} \in Y^{\prime}$, and $c \in \mathbb{F}$. Then, there exists a unique bilinear mapping $v$ from $X^{\prime} \times Y^{\prime}$ to $\mathbb{F}$ such that

$$
|u(f, g)-v(f, g)| \leq \tilde{\varphi}(f, g) \quad\left(f \in X^{\prime}, g \in Y^{\prime}\right) .
$$

Moreover, if the mapping $u$ satisfies

$$
|| u(f, g)|-| f(x) g(y)|| \leq \varphi(f, g)
$$

for some fixed $x \in X$ and $y \in Y$, then $\|v\|=\|x \otimes y\|$ and so in particular $v$ is bounded. 
Proof. Putting $c=1$ and replacing $f_{1}$ and $f_{2}$ in (2.3) by $f$ and dividing both sides by 2 , we get

$$
\left|\frac{1}{2} u(2 f, g)-u(f, g)\right| \leq \frac{1}{2} \varphi_{1}(f, f, g)
$$

for all $f \in X^{\prime}$ and $g \in Y^{\prime}$. Replacing $f$ by $2 f$ in (2.7) and dividing both sides by 2 , we find that

$$
\left|\frac{1}{2^{2}} u\left(2^{2} f, g\right)-\frac{1}{2} u(2 f, g)\right| \leq \frac{1}{2^{2}} \varphi_{1}(2 f, 2 f, g)
$$

for all $f \in X^{\prime}$ and $g \in Y^{\prime}$. Combining (2.7) with (2.8), we obtain

$$
\left|\frac{1}{2^{2}} u\left(2^{2} f, g\right)-u(f, g)\right| \leq \frac{1}{2} \varphi_{1}(f, f, g)+\frac{1}{2^{2}} \varphi_{1}(2 f, 2 f, g)
$$

for all $f \in X^{\prime}$ and $g \in Y^{\prime}$. By induction on $n$, we conclude that

$$
\left|\frac{1}{2^{n}} u\left(2^{n} f, g\right)-u(f, g)\right| \leq \sum_{i=0}^{n-1} \frac{1}{2^{i+1}} \varphi_{1}\left(2^{i} f, 2^{i} f, g\right)
$$

for all $f \in X^{\prime}$ and $g \in Y^{\prime}$. We now turn to use the Cauchy convergence criterion. Replace $f$ by $2^{k} f$ in (2.9) and divide both sides by $2^{k}$, where $k$ is an arbitrary positive integer, to get

$$
\left|\frac{1}{2^{n+k}} u\left(2^{n+k} f, g\right)-\frac{1}{2^{k}} u\left(2^{k} f, g\right)\right| \leq \sum_{i=k}^{n+k-1} \frac{1}{2^{i+1}} \varphi_{1}\left(2^{i} f, 2^{i} f, g\right)
$$

for all $f \in X^{\prime}, g \in Y^{\prime}$, and all positive integers $n \geq k$. It follows from the last inequality and (2.1) that the sequence $\left\{\frac{1}{2^{n}} u\left(2^{n} f, g\right)\right\}$ is a Cauchy sequence for all $f \in X^{\prime}$ and $g \in Y^{\prime}$. Since $\mathbb{F}$ is a complete field, this sequence converges. Define $v(f, g):=\lim _{n \rightarrow \infty} \frac{1}{2^{n}} u\left(2^{n} f, g\right)$. Taking the limit as $n \rightarrow \infty$ in (2.9), we find that the inequality (2.5) holds for all $f \in X^{\prime}$ and $g \in Y^{\prime}$. Replace $f_{1}$ and $f_{2}$ in (2.3) by $2^{n} f_{1}$ and $2^{n} f_{2}$ respectively and divide both sides by $2^{n}$ and take the limit as $n \rightarrow \infty$ and apply then (2.2) to get the mapping $f \longmapsto v(f, g)$ is linear. By a similar way one can replace $f$ in (2.4) by $2^{n} f$ and divide both sides by $2^{n}$ to deduce that the mapping $g \longmapsto v(f, g)$ is linear. Consequently, the mapping $v$ is bilinear. Our next claim is to prove that $v$ is unique. Let $v^{\prime}$ be another mapping satisfying (2.5). Hence,

$$
\begin{aligned}
\left|v(f, g)-v^{\prime}(f, g)\right| & =\frac{1}{2^{k}}\left|v\left(2^{k} f, g\right)-v^{\prime}\left(2^{k} f, g\right)\right| \\
& \leq \frac{2}{2^{k}} \tilde{\varphi}\left(2^{k} f, g\right) \\
& =2 \sum_{i=k}^{\infty} \frac{1}{2^{i+1}} \varphi_{1}\left(2^{i} f, 2^{i} f, g\right)
\end{aligned}
$$


for all $f \in X^{\prime}$ and $g \in Y^{\prime}$. Passing to the limit as $k \rightarrow \infty$, we conclude that $v$ is unique. Replace $f$ by $2^{n} f$ in (2.6) and divide both sides by $2^{n}$, to arrive at

$$
\left|\frac{1}{2^{n}}\right| u\left(2^{n} f, g\right)|-| f(x) g(y)|| \leq \frac{1}{2^{n}} \varphi\left(2^{n} f, g\right)
$$

for all $f \in X^{\prime}$ and $g \in Y^{\prime}$. Taking the limit as $n \rightarrow \infty$ in (2.10) and applying the definition of the norm, we conclude that $\|v\|=\|x \otimes y\|$ and so $v$ is bounded. by

Remark 1. Under the same hypotheses of Theorem 1, with (2.1) and (2.2) replaced

$$
\begin{gathered}
\tilde{\varphi}(f, g):=\sum_{i=0}^{\infty} \frac{1}{2^{i+1}} \varphi_{2}\left(f, 2^{i} g, 2^{i} g\right)<\infty, \\
\lim _{n \rightarrow \infty} \frac{1}{2^{n}} \varphi_{1}\left(f_{1}, f_{2}, 2^{n} g\right)=\lim _{n \rightarrow \infty} \frac{1}{2^{n}} \varphi_{2}\left(f, 2^{n} g_{1}, 2^{n} g_{2}\right)=\lim _{n \rightarrow \infty} \frac{1}{2^{n}} \varphi\left(f, 2^{n} g\right)=0,
\end{gathered}
$$

there exists a unique mapping $v \in \mathscr{B} \mathscr{L}\left(X^{\prime} \times Y^{\prime}, \mathbb{F}\right)$ satisfying (2.5). Note that by using (2.4) and the same method as in the proof of Theorem 1, we can define $v(f, g):=$ $\lim _{n \rightarrow \infty} \frac{1}{2^{n}} u\left(f, 2^{n} g\right)$.

In the following corollaries, as a consequence of Theorem 1, we show the Rassias stability of algebraic tensor products.

Corollary 1. Let $x \in X, y \in Y$, and $u: X^{\prime} \times Y^{\prime} \rightarrow \mathbb{F}$ be a mapping such that

$$
\begin{aligned}
\|u(f, g)|-| f(x) g(y)\| \leq \alpha+\beta\left(\|f\|^{p}+\right. & \left.\|g\|^{p}\right)+\gamma\|f\|^{p}\|g\|^{p}, \\
\left|u\left(c f_{1}+f_{2}, g\right)-c u\left(f_{1}, g\right)-u\left(f_{2}, g\right)\right| \leq \alpha & +\beta\left(\left\|f_{1}\right\|^{q}+\left\|f_{2}\right\|^{q}+\|g\|^{q}\right) \\
& +\gamma\left\|f_{1}\right\|^{\frac{q}{2}}\left\|f_{2}\right\|^{\frac{q}{2}}\|g\|^{q}, \\
\left|u\left(f, c g_{1}+g_{2}\right)-c u\left(f, g_{1}\right)-u\left(f, g_{2}\right)\right| \leq \alpha & +\beta\left(\|f\|^{r}+\left.\left\|g_{1}\right\|\right|^{r}+\left\|g_{2}\right\|^{r}\right) \\
& +\gamma\|f\|^{r}\left\|g_{1}\right\|^{\frac{r}{2}}\left\|g_{2}\right\|^{\frac{r}{2}}
\end{aligned}
$$

for all $f, f_{1}, f_{2} \in X^{\prime}, g, g_{1}, g_{2} \in Y^{\prime}$, and $c \in \mathbb{F}$, where $p, q, r, \alpha, \beta$, and $\gamma$ are constants with $0 \leq p, q, r<1, \alpha>0$, and $\beta, \gamma \geq 0$. Then, there exists a unique mapping $v \in \mathscr{B} \mathscr{L}\left(X^{\prime} \times Y^{\prime}, \mathbb{F}\right)$ such that $\|v\|=\|x \otimes y\|$ and

$$
|u(f, g)-v(f, g)| \leq \alpha+\beta\left(2 k\|f\|^{q}+\|g\|^{q}\right)+\gamma k \mid f\left\|^{q}\right\| g \|^{q}
$$

for all $f \in X^{\prime}$ and $g \in Y^{\prime}$, where $k=\frac{1}{2-2^{q}}$.

Remark 2. Under the hypotheses of Corollary 1 and using Remark 1, there exists a unique mapping $v \in \mathscr{B} \mathscr{L}\left(X^{\prime} \times Y^{\prime}, \mathbb{F}\right)$ such that $\|v\|=\|x \otimes y\|$ and

$$
|u(f, g)-v(f, g)| \leq \alpha+\beta\left(\|f\|^{r}+2 k\|g\|^{r}\right)+\gamma k \mid f\left\|^{r}\right\| g \|^{r}
$$

for all $f \in X^{\prime}$ and $g \in Y^{\prime}$, where $k=\frac{1}{2-2^{r}}$. 
Theorem 2. Let $\left\{x_{i}\right\}_{i=1}^{m}$ and $\left\{y_{i}\right\}_{i=1}^{m}$ be linearly independent sets in $X$ and $Y$ respectively and $u$ be a mapping from $X^{\prime} \times Y^{\prime}$ to $\mathbb{F}$ for which there exist mappings $\varphi_{1}$ : $X^{\prime} \times X^{\prime} \times Y^{\prime} \longrightarrow \mathbb{R}^{+}, \varphi_{2}: X^{\prime} \times Y^{\prime} \times Y^{\prime} \longrightarrow \mathbb{R}^{+}$, and $\varphi: X^{\prime} \times Y^{\prime} \longrightarrow \mathbb{R}^{+}$satisfying (2.1), (2.2), (2.3), (2.4) and

$$
|| u(f, g)\left|-\sum_{i=1}^{m}\right| f\left(x_{i}\right) g\left(y_{i}\right)|| \leq \varphi(f, g)
$$

for all $f \in X^{\prime}, g \in Y^{\prime}$. Then, there exists a unique mapping $v \in \mathscr{B} \mathscr{L}\left(X^{\prime} \times Y^{\prime}, \mathbb{F}\right)$ such that

$$
|u(f, g)-v(f, g)| \leq \tilde{\varphi}(f, g) \quad\left(f \in X^{\prime}, g \in Y^{\prime}\right), \quad\|v\| \leq \sum_{i=1}^{m}\left\|x_{i} \otimes y_{i}\right\| .
$$

In the following our interest is to provide a dual for Theorem 1.

Theorem 3. Let $x \in X, y \in Y$, and let $u: X^{\prime} \times Y^{\prime} \rightarrow \mathbb{F}$ be a mapping for which there exist mappings $\varphi_{1}: X^{\prime} \times X^{\prime} \times Y^{\prime} \longrightarrow \mathbb{R}^{+}, \varphi_{2}: X^{\prime} \times Y^{\prime} \times Y^{\prime} \longrightarrow \mathbb{R}^{+}$, and $\varphi$ : $X^{\prime} \times Y^{\prime} \longrightarrow \mathbb{R}^{+}$satisfying (2.3), (2.4), (2.6), and

$$
\tilde{\varphi}(f, g):=\sum_{i=0}^{\infty} 2^{i} \varphi_{1}\left(\frac{f}{2^{i+1}}, \frac{f}{2^{i+1}}, g\right)<\infty,
$$

$$
\lim _{n \rightarrow \infty} 2^{n} \varphi_{1}\left(\frac{f_{1}}{2^{n}}, \frac{f_{2}}{2^{n}}, g\right)=\lim _{n \rightarrow \infty} 2^{n} \varphi_{2}\left(\frac{f}{2^{n}}, g_{1}, g_{2}\right)=\lim _{n \rightarrow \infty} 2^{n} \varphi\left(\frac{f}{2^{n}}, g\right)=0
$$

for all $f, f_{1}, f_{2} \in X^{\prime}, g, g_{1}, g_{2} \in Y^{\prime}$. Then, there exists a unique mapping $v \in$ $\mathscr{B} \mathscr{L}\left(X^{\prime} \times Y^{\prime}, \mathbb{F}\right)$ satisfying (2.5).

Proof. By induction on $n$, we conclude that

$$
\left|u(f, g)-2^{n} u\left(\frac{f}{2^{n}}, g\right)\right| \leq \sum_{i=0}^{n-1} 2^{i} \varphi_{1}\left(\frac{f}{2^{i+1}}, \frac{f}{2^{i+1}}, g\right)
$$

for all $f \in X^{\prime}$ and $g \in Y^{\prime}$. Replace $f$ by $\frac{f}{2^{k}}$ in (2.19) and multiply both sides by $2^{k}$, where $k$ is an arbitrary positive integer, to get

$$
\left|2^{k} u\left(\frac{f}{2^{k}}, g\right)-2^{n+k} u\left(\frac{f}{2^{n+k}}, g\right)\right| \leq \sum_{i=k}^{n+k-1} 2^{i} \varphi_{1}\left(\frac{f}{2^{i+1}}, \frac{f}{2^{i+1}}, g\right)
$$

for all $f \in X^{\prime}, g \in Y^{\prime}$, and all positive integers $n \geq k$. In order to use the Cauchy convergence criterion, the last inequality and (2.17) imply the sequence $\left\{2^{n} u\left(\frac{f}{2^{n}}, g\right)\right\}$ is a Cauchy sequence for all $f \in X^{\prime}$ and $g \in Y^{\prime}$. Due to completeness of $\mathbb{F}$, this sequence converges. Define $v(f, g):=\lim _{n \rightarrow \infty} 2^{n} u\left(\frac{f}{2^{n}}, g\right)$. Taking the limit as $n \rightarrow$ $\infty$ in (2.19), we deduce that the inequality (2.5) holds for all $f \in X^{\prime}$ and $g \in Y^{\prime}$. The rest of the proof is similar to that of Theorem 1 . 
Remark 3. Under the same hypotheses of Theorem 3, with (2.17) and (2.18) replaced by

$$
\tilde{\varphi}(f, g):=\sum_{i=0}^{\infty} 2^{i} \varphi_{2}\left(f, \frac{g}{2^{i+1}}, \frac{g}{2^{i+1}}\right)<\infty,
$$

$$
\lim _{n \rightarrow \infty} 2^{n} \varphi_{1}\left(f_{1}, f_{2}, \frac{g}{2^{n}}\right)=\lim _{n \rightarrow \infty} 2^{n} \varphi_{2}\left(f, \frac{g_{1}}{2^{n}}, \frac{g_{2}}{2^{n}}\right)=\lim _{n \rightarrow \infty} 2^{n} \varphi\left(f, \frac{g}{2^{n}}\right)=0,
$$

there exists a unique mapping $v \in \mathscr{B} \mathscr{L}\left(X^{\prime} \times Y^{\prime}, \mathbb{F}\right)$ satisfying (2.5). We remark that by using (2.4) and the same method as in the proof of Theorem 3, one can define $v(f, g):=\lim _{n \rightarrow \infty} 2^{n} u\left(f, \frac{g}{2^{n}}\right)$.

Corollary 2. Let $x \in X, y \in Y$, and $u: X^{\prime} \times Y^{\prime} \rightarrow \mathbb{F}$ be a mapping such that

$$
\begin{gathered}
\left|\|u(f, g)|-| f(x) g(y)\| \leq \alpha\|f\|^{p}\|g\|^{p},\right. \\
\left|u\left(c f_{1}+f_{2}, g\right)-c u\left(f_{1}, g\right)-u\left(f_{2}, g\right)\right| \leq \beta\left\|f_{1}\right\|^{\frac{q}{2}}\left\|f_{2}\right\|^{\frac{q}{2}}\|g\|^{q}, \\
\left|u\left(f, c g_{1}+g_{2}\right)-c u\left(f, g_{1}\right)-u\left(f, g_{2}\right)\right| \leq \gamma\|f\|^{r}\left\|g_{1}\right\|^{\frac{r}{2}}\left\|g_{2}\right\|^{\frac{r}{2}}
\end{gathered}
$$

for all $f, f_{1}, f_{2} \in X^{\prime}, g, g_{1}, g_{2} \in Y^{\prime}$, and $c \in \mathbb{F}$, where $p, q, r>1$, and $\alpha, \beta, \gamma>0$. Then, there exists a unique mapping $v \in \mathscr{B} \mathscr{L}\left(X^{\prime} \times Y^{\prime}, \mathbb{F}\right)$ such that $\|v\|=\|x \otimes y\|$ and

$$
|u(f, g)-v(f, g)| \leq \frac{\beta}{2^{q}-2}\|f\|^{q}\|g\|^{q}\left(f \in X^{\prime}, g \in Y^{\prime}\right) .
$$

Proof. It is enough to define $\varphi(f, g):=\alpha\|f\|^{p}\|g\|^{p}$, $\varphi_{1}\left(f_{1}, f_{2}, g\right):=\beta\left\|f_{1}\right\|^{\frac{q}{2}}\left\|f_{2}\right\|^{\frac{q}{2}}\|g\|^{q}$, and $\varphi_{2}\left(f, g_{1}, g_{2}\right):=\gamma\|f\|^{r}\left\|g_{1}\right\|^{\frac{r}{2}}\left\|g_{2}\right\|^{\frac{r}{2}}$ for all $f, f_{1}, f_{2} \in X^{\prime}$ and $g, g_{1}, g_{2} \in Y^{\prime}$ and then apply Theorem 3 .

Remark 4. Under the hypotheses of Corollary 2 and using Remark 3, there exists a unique mapping $v \in \mathscr{B} \mathscr{L}\left(X^{\prime} \times Y^{\prime}, \mathbb{F}\right)$ such that $\|v\|=\|x \otimes y\|$ and

$$
|u(f, g)-v(f, g)| \leq \frac{\gamma}{2^{r}-2}\|f\|^{r}\|g\|^{r}\left(f \in X^{\prime}, g \in Y^{\prime}\right) .
$$

Theorem 4. Let $\left\{x_{i}\right\}_{i=1}^{m}$ and $\left\{y_{i}\right\}_{i=1}^{m}$ be linearly independent sets in $X$ and $Y$ respectively and $u$ be a mapping from $X^{\prime} \times Y^{\prime}$ to $\mathbb{E}$ for which there exist mappings $\varphi_{1}$ : $X^{\prime} \times X^{\prime} \times Y^{\prime} \longrightarrow \mathbb{R}^{+}, \varphi_{2}: X^{\prime} \times Y^{\prime} \times Y^{\prime} \longrightarrow \mathbb{R}^{+}$, and $\varphi: X^{\prime} \times Y^{\prime} \longrightarrow \mathbb{R}^{+}$satisfying (2.17), (2.18), (2.15), (2.3), (2.4). Then, there exists a unique mapping $v \in \mathscr{B} \mathscr{L}\left(X^{\prime} \times\right.$ $\left.Y^{\prime}, \mathbb{F}\right)$ satisfying $(2.16)$.

\section{ACKNOWLEDGEMENT}

The authors would like to thank the anonymous referee for his/her valuable comments concerning the statement of Theorem 1. 


\section{REFERENCES}

[1] T. Aoki, "On the stability of the linear transformation in Banach spaces," J. Math. Soc. Japan., vol. 2, pp. 64-66, 1950.

[2] R. Badora, “On approximate derivations," Math. Inequal. Appl., vol. 9, pp. 167-173, 2006.

[3] F. F. Bonsal and J. Duncan, Complete normed algebra. New York, Heidelberg, Berlin: SpringerVerlag, 1973.

[4] V. A. Faizev, T. M. Rassias, and P. K. Sahoo, "The space of $(\psi, \gamma)$-additive mappings on semigroups," Trans. Amer. Math. Soc., vol. 354, no. 11, pp. 4455-4472, 2002.

[5] P. Gavruta, "Generalization of the Hyers-Ulam-Rassias stability of approximately additive mappings," J. Math. Anal. Appl., vol. 184, pp. 431-436, 1994.

[6] P. M. Gruber, "Stability of isometries," Trans. Amer. Math. Soc., vol. 245, pp. 263-277, 1978.

[7] D. H. Hyers, "On the stability of the linear functional equation," Proc. Nat. Acad. Sci. U.S.A., vol. 27, pp. 222-224, 1941.

[8] D. H. Hyers, G. Isac, and T. M. Rassias, "On the asymptoticity aspect of Hyers-Ulam stability of mappings," Proc. Amer. Math. Soc., vol. 162, no. 2, pp. 425-430, 1998.

[9] D. H. Hyers, G. Isac, and T. M. Rassias, Stability of functional equations in several variables. Boston: Birkhauser, 1998.

[10] D. H. Hyers and T. M. Rassias, "Approximate homomorphisms," Aequations Mathematicae, vol. 44, pp. 125-153, 1992.

[11] T. Miura, S. E. Takahashi, and G. Hirasawa, "Hyers-Ulam-Rassias stability of Jordan homomorphisms on Banach algebras," J. Inequal. Appl., vol. 1, pp. 435-441, 2004

[12] M. S. Moslehian and T. M. Rassias, "Stability of functional equations in non-Archimedean spaces," Applicable Analysis and Discrete Mathematics, vol. 1, no. 2, pp. 325-334, 2007.

[13] A. Najati and T. M. Rassias, "Stability of a mixed functional equation in several variables on Banach modules," Nonlinear Analysis, vol. 72, no. 3-4, pp. 1755-1767, 2010.

[14] A. Prastaro and T. M. Rassias, "Ulam stability in geometry of PDE's," Nonlinear Func. Anal. Appl., vol. 8, no. 2, pp. 259-278, 2003.

[15] T. M. Rassias, "On a modified Hyers-Ulam sequence," J. Math. Anal. Appl., vol. 158, pp. 106-113, 1991.

[16] T. M. Rassias, "On the stability of the linear mapping in Banach spaces," Proc. Amer. Math. Soc., vol. 72, pp. 297-300, 1998.

[17] T. M. Rassias, "On the stability of functional equations and a problem of Ulam," Acta. Appl. Math., vol. 62 , no. 1 , pp. $23-130,2000$.

[18] T. M. Rassias, "On the stability of functional equations in Banach spaces," J. Math. Anal. Appl., vol. 251, no. 1, pp. 264-284, 2000.

[19] T. M. Rassias, "The problem of S. M. Ulam for approximately multiplicative mappings," J. Math. Anal. Appl., vol. 246, no. 2, pp. 352-378, 2000.

[20] T. M. Rassias and P. Semrl, "On the Hyers-Ulam stability of linear mappings," J. Math. Anal. Appl., vol. 173, pp. 325-338, 1993.

[21] T. M. Rassias, "On the stability of functional equations originated by a problem of Ulam," Mathematica, vol. 44, no. 1, pp. 39-75, 2002.

[22] S. M. Ulam, A collection of mathematical problems. New York: Interscience Publisher, 1960.

Authors' addresses

\section{Ismail Nikoufar}

Department of Mathematics, Payame Noor University, P.O. BOX 19395-3697 Tehran, Iran

E-mail address: nikoufar@pnu.ac.ir 
Themistocles M. Rassias

Department of Mathematics, National Technical University of Athens, Zografou, Campus 15780 Athens, Greece

E-mail address: trassias@math.ntua.gr 